\title{
THERMODYNAMICS OF COMPLEX SYSTEMS
}

\section{(An Introduction to Ecophysics)}




\section{SERIES ON ADVANCES IN STATISTICAL MECHANICS}

Editor-in-chief: M. Rasetti

\section{Published}

Vol. 1: Integrable Systems in Statistical Mechanics edited by G. M. D'Ariano, A. Montorsi and M. Rasetti

Vol. 2: Modern Methods in Equilibrium Statistical Mechanics by M. Rasetti

Vol. 3: Progress in Statistical Mechanics edited by C. K. Hu

Vol. 6: New Problems, Methods and Techniques in Quantum Field Theory and Statistical Mechanics edited by $M$. Rasetti

Forthcoming

Vol. 5: Potts Model and Related Problems in Statistical Mechanics by $P$. Martin 
Series on Advances in Statistical Mechanics - Vol.4

\title{
THERMODYNAMICS OF COMPLEX SYSTEMS
}

\section{(An Introduction to Ecophysics)}

\author{
Luigi Sertorio \\ Department of Theoretical Physics \\ University of Torino, ITALY
}




\section{Published by}

World Scientific Publishing Co. Pte. Ltd.

P O Box 128, Farrer Road, Singapore 9128

USA office: 687 Hartwell Street, Teaneck, NJ 07666

UK office: 73 Lynton Mead, Totteridge, London N20 8DH

Library of Congress Cataloging-in-Publication data is available.

\section{THERMODYNAMICS OF COMPLEX SYSTEMS}

Copyright $\odot 1991$ by World Scientific Publishing Co. Pte. Ltd.

All rights reserved. This book, or parts thereof, may not be reproduced in any form or by any means, electronic or mechanical, including photocopying, recording or any information storage and retrieval system now known or to be invented, without written permission from the Publisher.

ISBN $9971-50-978-4$

Printed in Singapore by JBW Printers \& Binders Pte. Ltd. 


\section{Introduction}

Almost everything that happens around us are complex, non-linear, dissipative events. Fire, wind, the clouds, a jet of water, the crashing of a vase, an athletic performance; and within us, our memory. Non-dissipative, Hamiltonian events too are complex and non-linear.

If there is one thing that is very difficult to understand, and little investigated, it is why Nature has simple laws that can be confirmed in simple cases and which can nearly always be dealt with linear differential equations. Nature organizes the masses of astronomical bodies in a prevalently spherical symmetry which allows a very simple calculation of the field of gravity, something which is generally incalculable. The gravitational interactions, which are non-linear and intractable in cases of three bodies or more, are organised so that the multi-body problems are dominated by a prevailing mass that allows the perturbative method to be applied.

Why is linearity important? The virtues of linear differential equations are really manifold. Linear regimes are those whereby double cause produces double effect (the solution is linear in the known term). Moreover, the imperfect measurement of an event does not prevent one from gathering its main properties, and in its turn, this fact produces the property that the experiment can be easily repeated (weak dependence on initial conditions). The simplified model contains the properties of the more accurate description (this is the property by which the kinds of behaviour of the linear equations do not vary with the dimension of the phase space, i.e. of the number of unknown functions treated).

Why is it that these wonderful properties exist and that we are so used to them that we no longer think of discussing them critically? We can give this tentative reply: the events that surround us belong to a physical Universe in near equilibrium; dynamic properties in the proximity of states of equilibrium are well described by linear approximation and this is particularly true in the case of dissipative systems. There is no doubt that with simple cases and linear approximations, we are observing some very profound aspects of Nature.

So what is the point of tackling complex events, which are by definition far from equilibrium, and almost always non-linear?

This book tries to answer this question with a step-by-step approach.

The first four sections of the book comprise a summary of equilibrium thermodynamics, i.e. the study of equilibrium and near equilibrium. In this 
framework disequilibrium remains unexplained; the disequilibrium can be created by the experimenter, who manipulates events. This is the traditional view of equilibrium thermodynamics. However disequilibrium is a global datum of Nature, in fact the most profound of all, as it derives from the cosmological history of the present Universe (sections 6, 9). The disequilibrium between the stars and the cold vacuum is a situation of stationary disequilibrium that is characterised by extremely long time scales (the eternity of the ancient thinkers) and creates the situation of the cosmological engine mentioned in section 9 . Therefore, we have a non-linear, dissipative, complex system that cannot be classified into the categories of simple solutions we are used to - the ecosystem Earth.

The formalism describing complex systems is that of non-equilibrium thermodynamics, which is a rather difficult theory. The fundamental equations are derived in sections 10-12 using a line of reasoning that may be recapitulated in a few words as follows. In equilibrium thermodynamics there are two developments from the theoretical roots: treating only purely dissipative phenomena, the natural ones; or emphasizing also Carnot's reversible cycles, the ones which produce work. The second is the engineers' route. Having understood that the cosmological engine exists, the engineers' route now acquires fundamental value. It is for this reason that in sections 10-12 the formulation of the fundamental equations has been made developing Gibbs' concept of availability and thereby expressing the dissipative and active systems in a unified way. This also allows us to neatly arrange an old idea of Tolman in 1948, which was not fully appreciated at the time.

The derivation of these sections covers only homogeneous systems, that is to say with only one chemical species. This is a serious limitation. In fact the interaction between the Physical Ecosystem and the dynamics of living species is predominantly of chemical thermodynamics. There is no justification for this omission: the generalization to heterogeneous systems is necessary. Remaining in the domain of homogeneous systems, a simplified, albeit complex, model is discussed that allows us to estimate the energy efficiency of the Earth, which is a sort of Carnot super engine. The ideal efficiency is minute, in order of $10^{-3}$, as shown in section 16 . Thus the processed power is $10^{-3}$ times the solar input. Living organisms are in equilibrium with the thermodynamic ecosystem (they do not use fossil energy like man) and therefore their thermodynamics is a continuous processing of information, something that can be measured in a few $\frac{W \text { att }}{K^{0} m^{3}} \cdot 10^{-2}$. Here we are speaking of physicists' real entropy. 
Every adventure in scientific thought must be able to say something to man: what use is this to me, what am I learning from this? Now man is that animal who to do the same things as other living beings, i.e. to keep processing information, uses energy-consuming machines. Just to give a concrete example, every American processes on the average $10 \mathrm{~kW}=10^{4}$ Watt to do what he or she does: loves, hates, works, goes on holiday: the natural reference number is the one found in section 16 , namely about $\frac{1 W \text { att }}{m^{3}}$. It is interesting to wonder how much of this enormous quantity of power/ person is rapidly dissipated, instead of creating intelligent structures. Certainly this is a huge problem that will interest researchers for decades to come.

However there is a problem which non-equilibrium thermodynamics can teach us something about immediately. Quite rightly man likes to live in thermostable environments, but his Earth's ecosystem is not stable and pleasant everywhere. Man obtains what he wants in a dissipative way by heating and cooling with machines. On the other hand he could heat and cool by manipulating the disequilibria of our ecosystem. Thus just as in general the disequilibria can be a source of work, they can also be a source of thermostabilising power. This is what is discussed in the last part of the book. 
This page is intentionally left blank 


\section{Acknowledgments}

I wish to thank Peter Carruthers, John Kessler, Gordon MacDonald, Arnaldo Longhetto, Adriano Zecchina, for their interest and help; Elliot Leader, Jeanette Nelson, Albert Werbrouck for critical reading of the text. The merit for the drawings goes to Laura Socci. I am grateful to Lucy Carruthers, Magda Spedini and Paola Zalone for their generous collaboration and computer assistance. 
This page is intentionally left blank 


\section{Index}

Introduction $\quad \mathbf{v}$

Acknowledgments ix

1. 1583; The harmonic oscillator. 1

2. Irreversibility is not to be deduced. 4

3. Equilibrium thermodynamics. Irreversibility and $\begin{array}{ll}\text { reversible processes. } & 7\end{array}$

4. Equilibrium thermodynamics. Irreversibility and disequilibrium. 19

5. From laboratory to environment. 26

6. Expansion, large scale uniformity and local disequilibrium. 29

$\begin{array}{ll}\text { 7. Reversible, irreversible. } & 37\end{array}$

8. Time scales. 61

9. The cosmological engine. 68

10. The equations of non-equilibrium thermodynamics.

$\begin{array}{ll}\text { Rigid systems. } & 69\end{array}$

11. The equations of non-equilibrium thermodynamics.

$\begin{array}{ll}\text { Fluid systems. } & 80\end{array}$

12. Active systems. 94

13. Ecosystems. 100

14. Ecosystem Earth and the radiation fluxes. 104

15. Ecosystem Earth and its constitution. 107

16. A model for the evaluation of Earth's availability. 109

17. Comments on section $16 . \quad 125$

18. Interacting with the ecosystem. Global warming. 127

19. Control and information. 146

20. Brief sketch of one-directional control systems. 148

21. Remarks on global control systems. 158

22. Geophysical inputs. 161

23. Thermodynamics of the inert house. 168

24. Variable structure and online automaton $\mathrm{Z}(4,3)$. 179

25. Thermodynamic demon and Entropy. 191

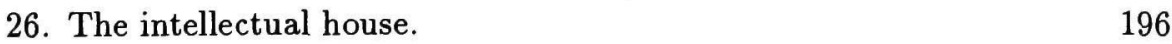

27. Conclusions. 206 